\title{
Metabolic syndrome and insulin resistance in migraine
}

\author{
Sanjeev K. Bhoi · Jayantee Kalita $\cdot$ Usha K. Misra
}

Received: 2 November 2011 / Accepted: 16 January 2012/Published online: 26 January 2012

(C) The Author(s) 2012. This article is published with open access at Springerlink.com

\begin{abstract}
Metabolic syndrome is associated with migraine but there is no study comparing the characteristics of migraine with and without metabolic syndrome from Southeast Asia. This study was therefore undertaken to compare the clinical characteristics of migraine in patients with and without metabolic syndrome and insulin resistance. 135 consecutive patients with migraine diagnosed on the basis of International Headache Society criteria were subjected to clinical evaluation as per fixed protocol. Headache severity, frequency and functional disability were recorded. Metabolic syndrome was diagnosed as per National Cholesterol Education Programme: Adult Treatment Panel III and International Diabetic Federation criteria. Insulin resistance was calculated by homeostases model assessment. Their age ranged between 14 and 61 years and 108 were females. Metabolic syndrome was present in $31.9 \%$ patients and only 13 were obese. Insulin resistance was present in $11.1 \%$. Metabolic syndrome was correlated with age, gender, number of triggers, years of headache and duration of migraine attacks. Insulin resistance correlated with duration of migraine attacks. From this study, it can be concluded that metabolic syndrome was present in $31.9 \%$ of the migraineurs which was mainly in elderly who had longer duration of headache and multiple triggers.
\end{abstract}

Keywords Migraine $\cdot$ Obesity $\cdot$ Metabolic syndrome Insulin resistance $\cdot$ HOMA

\footnotetext{
S. K. Bhoi · J. Kalita · U. K. Misra (ه)

Department of Neurology, Sanjay Gandhi Post Graduate Institute of Medical Sciences, Raebareily Road, Lucknow 226014, India e-mail: drukmisra@rediffmail.com; ukmisra@sgpgi.ac.in

J. Kalita

e-mail: jkalita@yahoo.com
}

\section{Introduction}

Migraine is a common disorder with overall prevalence of $5-15 \%$ [1-3].The prevalence of migraine in females is higher $(6-22 \%)$ compared to males $(3-7 \%)$ [4-6]. The pathogenesis of migraine is multifactorial and a number of genetic and environmental factors have been suggested. Recently, association of migraine with metabolic syndrome and insulin resistance has been reported [7, 8]. The prevalence of migraine was estimated as $11.9 \%$ in males and $22.5 \%$ in females with metabolic syndrome. Of the components of metabolic syndrome diabetes mellitus, increased waist circumference and body mass index (BMI) were more frequent in migraine patients compared to those without migraine. The frequency of hypertension and dyslipidemia, however, was not significantly different [9]. In a population-based study on obesity and migraine evaluating 30,215 participants whose mean age was 38.4 years including $65 \%$ females, revealed lack of association of BMI with the prevalence of migraine but was associated with the frequency of headache attacks. In the normal weight group, $4.4 \%$ had $10-15$ headache days per month increasing to $5.8 \%$ in the overweight and $20.7 \%$ in the morbidly obese [10]. Majority of these studies are from the western countries where obesity is common. In a population-based study, 685 women aged 40-74 years were evaluated for relationship of BMI and migraine. The proportion of obesity (BMI $>30$ ) did not differ in the women with active migraine, inactive migraine and those who never experienced migraine. The frequency, duration and severity of migraine attacks also did not differ between obese and non-obese migraine patients [11].

On PubMed search using Keywords "Migraine" and "Metabolic syndrome/insulin resistance/insulin metabolism" revealed six articles; two on metabolic syndrome and 
four on insulin metabolism in migraine $[8,9,12-15]$. The incidence of obesity is lower in Asian countries and we could not find any study from Southeast Asia evaluating the role of metabolic syndrome in migraine. In the present study, we report the frequency of metabolic syndrome in migraine patients and compare the clinical characteristics of migraine in patients with and without metabolic syndrome as well as insulin resistance.

\section{Patients and methods}

The patients with migraine were recruited from the neurology out patient service of Sanjay Gandhi Postgraduate Institute, Lucknow, India during January 2009-June 2010. The diagnosis of migraine was based on International Headache Society Criteria [16]. The children below 13 years of age were excluded because ours is an adult neurology service. All the patients were subjected to medical history and clinical evaluation as per a fixed protocol. Family history of headache in three generations was noted. Migraine triggers were enquired using a questionnaire evaluating various exogenous (sun exposure, noise, cold, hot, weather change, travelling, hair wash, dry hair, applying hair oil and food items) and endogenous triggers (physical and mental stress, fasting for $12 \mathrm{~h}$ or more, hunger, sleep deprivation and menstruation). The presence of mechanical (static and dynamic) and thermal allodynia was recorded from checklist which included combing hair, fan air, shower, shaving, resting head on pillow, wearing neck tie, ornaments, hair clip, scurf, veil and ear ring, hot and cold during the interview. The extent of allodynia was categorized into cephalic only and extension to extracephalic region (neck and upper limb). The duration of migraine in years, its frequency per month, associated symptoms, functional disability, duration of each attack in days and response to treatment were evaluated. The severity of headache was graded on a $0-3$ scale $(0=$ normal, $1=$ mild, $2=$ moderate, $3=$ severe headache). We considered average severity of headache in the last month. The functional disability was graded on $0-4$ scale $[0=$ normal, $1=$ mild impairment of activities of daily living (ADL), $2=$ moderate, $3=$ severe and $4=$ inability to perform ADL activities or bedridden state]. Presence of aura and associated symptoms such as nausea, vomiting, photophobia and phonophobia were also evaluated. The migraine index was calculated by average number of attacks per month multiplied by average severity of headache. Corrected migraine index was calculated by migraine index multiplied by average duration of attack.

Diagnosis of metabolic syndrome was based on National Cholesterol Education Programme (NCEP): Adult Treatment Panel (ATP) III 2001 [17] and International Diabetic
Federation (IDF) criteria [18], according to which three of the following criteria were needed including central obesity.

1. Central obesity: waist circumference $>90 \mathrm{~cm}$ in males and $80 \mathrm{~cm}$ in females.

2. Hypertriglyceridemia $>150 \mathrm{mg} / \mathrm{dl}$ or on specific medication.

3. Low HDL cholesterol $<40 \mathrm{mg} / \mathrm{dl}$ in males and $<50 \mathrm{mg} / \mathrm{dl}$ in females.

4. Hypertension: blood pressure $>130$ systolic or $>85 \mathrm{~mm} \mathrm{Hg}$ diastolic or specific medication.

5. Fasting plasma glucose $>100 \mathrm{mg} / \mathrm{dl}$ or on specific medication or previously diagnosed type 2 diabetes.

Insulin resistance and $\beta$ cell function were calculated in 90 patients by HOMA model [19].

HOMA-IR (insulin resistance)

$$
=\frac{\text { Fasting blood sugar } \times \text { fasting insulin }}{405}
$$

HOMA-B ( $\beta$ cell function) was calculated as follows :

$$
=\frac{360 \times \text { insulin }}{\text { Fasting blood sugar }}-63
$$

The cut-off value of insulin resistance was taken as $>1.77$ for the diagnosis of metabolic syndrome [20].

Blood counts, haemoglobin, ESR, platelet count, blood urea nitrogen, serum creatinine, bilirubin, transaminases and TSH were carried out. Fasting and 2-h post prandial blood sugar, lipid profile and insulin level were estimated. The blood sugar was assayed by autoanalyzer using oxidase-peroxidase method. The insulin was estimated by immunoradiometric assay using Siemens Medical Solutions Diagnostic, LA, USA. Patients with high thyroid stimulating hormone, renal and hepatic failure were excluded.

\section{Statistical analysis}

The migraine patients were categorized into those with metabolic syndrome and those without. The patients were also categorized into insulin resistant and those without based on HOMA-IR. The demographic, severity and frequency of migraine, presence of allodynia, aura and triggers were compared in those with and without metabolic syndrome as well as with and without insulin resistance using $X^{2}$ and independent $t^{\prime}$ test. The variables were considered significant if 2-tailed $p$ value was $<0.05$. The statistical analysis was done using SPSS 15 version software.

\section{Results}

There were 135 patients with migraine whose mean age was 31.4 (SD 10.5) years; 108 of whom were females. 
Table 1 Demography and clinical characteristics of migraine patients

\begin{tabular}{ll}
\hline Parameters & Number of patients $(n=135)$ \\
\hline Age (years) & $31.41 \pm 10.5$ (range 14-61) \\
Female & $108(80 \%)$ \\
Urban/rural & $81 / 54$ \\
Education (years) & $10.36 \pm 5.02$ \\
Family history of headache & $58(43 \%)$ \\
Total family members affected & $1.66 \pm 0.9$ (range $1-6)$ \\
Only patients & $77(57 \%)$ \\
1st generation & $33(24.4 \%)$ \\
2nd generation & $23(17 \%)$ \\
3 rd generation & $2(1.5 \%)$ \\
Migraine with aura & $7(5.2 \%)$ \\
Duration (years) & $8.48 \pm 6.7$ (range $0.5-40)$ \\
Frequency/months & $15.4 \pm 10.7$ (range 2-30) \\
Duration attack (days) & $1.8 \pm 0.8($ range $0.5-4)$ \\
Total no. of triggers & $10.84 \pm 2.78$ (range 2-15) \\
Allodynia & $116(85.9 \%)$ \\
Mechanical & $116(85.9 \%)$ \\
Static & $116(85.9 \%)$ \\
Dynamic & $115(85.2 \%)$ \\
Thermal & $27(20 \%)$ \\
Cephalic & $43(37.1 \%)$ \\
Extracephalic & $73(62.9 \%)$ \\
Number of metabolic parameters abnormality $(0-5)$ \\
0 & $11(8.1 \%)$ \\
1 & $41(30.4 \%)$ \\
2 & $40(29.6 \%)$ \\
3 & $26(19.3 \%)$ \\
4 & $14(10.4 \%)$ \\
5 & $3(2.2 \%)$ \\
\hline
\end{tabular}

Family history of migraine was present in 58 patients. The mean duration of headache was 8.5 (SD 6.7) years. Only seven patients had migraine with aura. The frequency of migraine ranged between 2 and 30 attacks per month; 54 of them had headache frequency of more than 15 attacks per month. Mechanical (static and dynamic) allodynia was present in $116(85.9 \%)$ and $27(20 \%)$ of them also had thermal allodynia. The allodynia was cephalic in 43 (37.1\%) and extended to extracephalic region in 73 (62.9\%) patients. The majority of patients [134 (99.3\%)] had moderate to severe headache. The general characteristics of the patients are provided in Table 1 .

Metabolic syndrome

The BMI was above 30 in $13(9.6 \%)$ patients and between 25 and 30 in 26 (19.3\%). Female patients were more overweight compared to males (34 vs. 5) and 2 females were morbidly obese $($ BMI $>35)$. Waist circumference was abnormal in $78(57.8 \%)$ patients. HDL was reduced in 108 (80\%) and triglyceride was increased in 28 (20.7\%) patients. Fasting blood sugar was high in $13(9.6 \%)$ patients. The systolic blood pressure was raised in 15 and diastolic in $33(24.5 \%)$ patients. Insulin resistance (HOMA-IR) was present in $10(11.1 \%)$ patients. $\beta$ cell function (HOMA-B) was $179.3 \pm 552.4$. Based on ATP-III and IDF criteria, 43 (31.9\%) patients had metabolic syndrome. Out of 5 components of metabolic syndromes, all 5 were present in 3, 4 in 14 and 3 in 26 patients. Only 2 components of metabolic syndrome were present in 40 patients, however they were not diagnosed as metabolic syndrome.

\section{Correlation}

The patients with metabolic syndrome were older $(p=$ $0.0001)$, more frequently females $(p=0.01)$, had longer duration of illness $(10.3 \pm 7.5$ vs. $7.6 \pm 6.2, p=$ $0.03)$, higher number of triggers $(11.6 \pm 2.5$ vs. $10.5 \pm$ $2.8, p=0.03)$ and longer duration of attack ( $2.0 \pm 0.8$ vs. $1.7 \pm 0.8, p=0.05)$ compared to those without metabolic syndrome. The majority of patients had multiple triggers; commonest being noise in $129(95.6 \%)$ followed by stress in $128(94.8 \%)$, sunlight in $121(89.6 \%)$ and fasting in 100 $(74.1 \%)$ patients. On comparing the triggers, head wash was more frequent in those with metabolic syndrome compared to without $(65.1 \%$ vs. $44.6 \% ; p=0.03)$. The details of triggers are given in Table 2 . The presence of aura ( 3 vs. $4, p=0.68)$, frequency of headache $(15.1 \pm$ 11.1 vs. $15.5 \pm 10.6, p=0.82)$, severity of migraine $(p=0.66)$, migraine index (MI) $(30.8 \pm 22.6$ vs. $37.0 \pm$ 28.0, $p=0.20$ ), corrected migraine index (MIc) (55.8 \pm 39.4 vs. $54.6 \pm 40.0, p=0.87)$, functional impairment $(p=0.19)$, presence of allodynia $(p=0.79)$ and extent of allodynia $(p=0.84)$ were not significantly different between the two groups (Table 3). Insulin resistance (HOMA-IR >1.77) was present in $10(11.1 \%)$ patients. There was no difference in demography, duration of migraine, migraine index, corrected migraine index, number of triggers, and fasting and hunger as triggers between the two groups (Table 4). The duration of migraine attack was more in the patients with insulin resistance $(p=0.04)$.

\section{Discussion}

In the present study, $31.9 \%$ of the migraine patients had metabolic syndrome but only $9.6 \%$ were obese. The diagnosis of metabolic syndrome was based on increased waist circumference (57.8\%), hypertriglyceridemia (20.7\%), hypertension $(27.4 \%)$, low HDL $(80.0 \%)$ and increased 
Table 2 Frequency of different triggers migraine and its comparison in the patients with and without metabolic syndrome
Table 3 Comparison of clinical characteristics in patients with and without metabolic syndrome

\begin{tabular}{lclll}
\hline Triggers & Total number of patients $(\%)$ & $\begin{array}{l}\text { Metabolic syndrome } \\
\text { Present } \\
N=43(31.9 \%)\end{array}$ & $\begin{array}{l}\text { Metabolic syndrome } \\
\text { Absent } \\
N=92(68.1 \%)\end{array}$ & $p$ value \\
\hline Physical stress & $128(94.8)$ & 41 & 87 & 1.00 \\
Mental stress & $128(94.8)$ & 40 & 88 & 0.70 \\
Fasting & $100(74.1)$ & 36 & 64 & 0.09 \\
Hunger & $100(74.1)$ & 36 & 64 & 0.09 \\
Sunlight & $121(89.6)$ & 41 & 80 & 0.22 \\
Noise & $129(95.6)$ & 43 & 86 & 0.18 \\
Cold exposure & $81(60)$ & 23 & 58 & 0.35 \\
Hot exposure & $100(74.1)$ & 34 & 66 & 0.41 \\
Weather change & $104(77)$ & 35 & 69 & 0.51 \\
Menstruation & $35(30.7)$ & 12 & 23 & 1.00 \\
Sleep deprivation & $119(88.1)$ & 40 & 79 & 0.27 \\
Food & $6(4.4)$ & 3 & 3 & 0.38 \\
Travel & $116(85.9)$ & 40 & 76 & 0.12 \\
Perfume & $33(24.4)$ & 11 & 22 & 0.83 \\
Hair oil & $3(2.2)$ & 1 & 2 & 1.00 \\
Head wash & $69(51.1)$ & 28 & 41 & 0.03 \\
Dry hair & $88(65.2)$ & 33 & 55 & 0.08 \\
\hline
\end{tabular}

\begin{tabular}{lllc}
\hline Parameters & $\begin{array}{l}\text { Metabolic syndrome } \\
\text { Present }\end{array}$ & $\begin{array}{l}\text { Metabolic syndrome } \\
\text { Absent } \\
N=43(31.9 \%)\end{array}$ & $\begin{array}{l}p \text { value } \\
\end{array}$ \\
\hline Age (years) & $37.8 \pm 7.7$ & $28.4 \pm 10.3$ & \\
Female & $40(93 \%)$ & $68(73.9 \%)$ & $<0.0001$ \\
Urban/rural & $26 / 17$ & $55 / 37$ & 0.01 \\
Education (years) & $9.35 \pm 4.76$ & $10.84 \pm 5.09$ & 1.00 \\
Family history of headache & $22(51.2 \%)$ & $36(39.1)$ & 0.11 \\
Total family members affected & $1.86 \pm 1.15$ & $1.57 \pm 0.79$ & 0.20 \\
Migraine with aura & $3(7 \%)$ & $4(4.4 \%)$ & 0.08 \\
Duration (years) & $10.28 \pm 7.5$ & $7.64 \pm 6.21$ & 0.68 \\
Total no. of triggers & $11.58 \pm 2.53$ & $10.49 \pm 2.84$ & 0.03 \\
Fasting & $36(83.7 \%)$ & $64(69.6 \%)$ & 0.03 \\
Hunger & $36(83.7 \%)$ & $64(69.6 \%)$ & 0.09 \\
Onset to peak time (min) & $32.09 \pm 32.68$ & $27.61 \pm 20.17$ & 0.09 \\
Frequency/months & $15.07 \pm 11.05$ & $15.52 \pm 10.58$ & 0.33 \\
Duration attack (days) & $2.0 \pm 0.75$ & $1.72 \pm 0.78$ & 0.82 \\
Severity of headache & $2.98 \pm 0.15$ & $2.93 \pm 0.29$ & 0.05 \\
Functional impairment & $3.28 \pm 0.7$ & $3.2 \pm 0.62$ & 0.37 \\
Rescue analgesics/mo & $13.07 \pm 9.82$ & $14.03 \pm 11.58$ & 0.48 \\
Allodynia & $38(88.4 \%)$ & $78(84.8 \%)$ & 0.64 \\
Migraine index (MI) & $30.75 \pm 22.59$ & $37.03 \pm 27.97$ & 0.79 \\
Corrected MI (MIc) & $55.83 \pm 39.43$ & $54.6 \pm 40.04$ & 0.20 \\
& & & 0.87
\end{tabular}

fasting blood sugar (9.6\%). Moreover, insulin resistance was present in $11.1 \%$ patients. These observations are in agreement with the reports from the western countries in which metabolic syndrome has been associated with migraine $[7,9,21]$. In our study, only 2 patients were morbidly obese and remaining 11 patients were obese. In a study from USA, the effect of BMI on the prevalence of severe headache or migraine revealed significant association of headache or migraine with low $\left(<18.5 \mathrm{~kg} / \mathrm{m}^{2}\right)$ and high BMI $\left(>30 \mathrm{~kg} / \mathrm{m}^{2}\right)$ [22]. In Asians, central obesity is 
Table 4 Comparison of clinical characteristics in patients with and without insulin resistance (HOMA-IR >1.775)

\begin{tabular}{llll}
\hline Parameters & $\begin{array}{l}\text { Insulin resistance } \\
\text { Present }\end{array}$ & $\begin{array}{l}\text { Insulin resistance } \\
\text { Absent } \\
N=10(11.1 \%)\end{array}$ & $\begin{array}{l}\text { N value } \\
\end{array}$ \\
\hline Age (years) & $33.0 \pm 9.04$ & $31.39 \pm 10.98$ & \\
Female & $8(80 \%)$ & $62(77.5 \%)$ & 0.66 \\
Urban/rural & $6 / 4$ & $45 / 35$ & 1.00 \\
Education (years) & $11.0 \pm 3.86$ & $10.05 \pm 5.06$ & 0.81 \\
Migraine with aura & $0(\%)$ & $6(7.5 \%)$ & 0.57 \\
Duration (years) & $9.98 \pm 6.30$ & $8.40 \pm 7.48$ & 0.37 \\
Total no. of triggers & $10.9 \pm 3.45$ & $11.15 \pm 2.54$ & 0.52 \\
Fasting & $7(70 \%)$ & $59(73.8 \%)$ & 0.78 \\
Hunger & $7(70 \%)$ & $59(73.8 \%)$ & 0.72 \\
Onset to peak & $28.0 \pm 13.78$ & $27.00 \pm 17.73$ & 0.72 \\
Severity of headache & $3.00 \pm 00$ & $2.95 \pm 0.27$ & 0.86 \\
Severity of functional impairment & $3.10 \pm 0.88$ & $3.16 \pm 0.63$ & 0.56 \\
Frequency/months & $16.00 \pm 12.27$ & $13.53 \pm 10.7$ & 0.78 \\
Duration attack (days) & $2.42 \pm 0.66$ & $1.90 \pm 0.78$ & 0.50 \\
Rescue analgesics/months & $17.10 \pm 14.43$ & $12.39 \pm 9.77$ & 0.04 \\
Allodynia & $9(90 \%)$ & $69(86.3 \%)$ & 0.18 \\
Family history of headache & $6(60 \%)$ & $31(38.6 \%)$ & 1.00 \\
Migraine index (MI) & $35.40 \pm 25.97$ & $31.46 \pm 25.98$ & 0.31 \\
Corrected MI (MIc) & $75.26 \pm 42.07$ & $52.65 \pm 43.01$ & 0.65 \\
No. of metabolic parameters & $3.70 \pm 1.16$ & $1.48 \pm 0.98$ & 0.12 \\
abnormality (metabolic syndrome) & & & $<0.0001$ \\
\hline
\end{tabular}

common and was present in $57.8 \%$ of our patients. Central obesity, high triglyceride and low HDL are reported to be the main component of metabolic syndrome in the Asians [23], whereas in the western countries high BMI is more common [13, 24].

Metabolic syndrome is associated with insulin resistance. The patients with migraine have significantly higher level of fasting glucose and insulin; both of which remain elevated after glucose loading suggesting insulin resistance [14]. Similar observations have also been reported in a smaller study in which oral glucose tolerance test and plasma glucose were significantly higher in migraine patients compared to controls. Insulin sensitivity is significantly altered in migraine patients as measured by ISIStumvoll and OGIS-180 index [8]. Insulin resistance is a state in which normal amount of insulin produces a subnormal physiological response. Insulin resistant state may increase free fatty acids and blood lipids which may induce migraine attack [25]. Low HDL is an important component of metabolic syndrome and HDL has anti-inflammatory property. Reduced LDL may reduce pain perception [26].

In our study, metabolic syndrome was significantly related to age. The mean age in the patients with metabolic syndrome was 37.8 (SD 7.7) years, whereas it was 28.4 (SD 10.3) years in those without. Age may be directly related to various components of metabolic syndrome such as obesity, hypertension, glucose intolerance and hyperlipidemia which may be an association rather than underlying mechanism. In our study, metabolic syndrome and migraine both were more common in females than males. Whether it is merely an association or it has a biological basis needs further study. Migraine is precipitated by a number of endogenous and exogenous triggers. Fasting and hunger are important triggers and have been reported in Indian subjects in $46.3 \%$ [27]. Fasting is common in both Hindus and Muslims as a religious practice. In the present study, the most common trigger was noise $(95.6 \%)$ followed by stress $(94.8 \%)$, sun exposure $(89.6 \%)$ and fasting (74.1\%). Fasting results in activation of insulin receptors which may trigger a migraine attack [28]. Low sucrose diets may reduce the frequency of migraine attack [29]. In migraineurs, the onset of diabetes mellitus increases the average yearly number of headache days [30]. In our study, fasting was neither associated with metabolic syndrome nor with insulin resistance but the duration of migraine attack (days) was significantly related to insulin resistance. Our results are limited by absence of a control group, absence of children, disproportionately large number of females and unequal number of patients with and without metabolic syndrome. In a population-based study from North India, the prevalence of metabolic syndrome is $67.2 \%$ (male $75.9 \%$ and female $58.4 \%$ ) and obesity in 
$79.3 \%$ (male $74.5 \%$ and female $84.0 \%$ ) [31]. The lower frequency of metabolic syndrome and obesity in our study compared to the above-mentioned study may be due to the referral bias. The study by Yadav et al. [31] was based on urban affluent population. We have studied migraine patients from different regions of North India including both urban and rural population comprising diverse socioeconomic classes.

Based on our results, it can be concluded that migraine is associated with metabolic syndrome in $31.9 \%$ and insulin resistance in $11.1 \%$ patients. The patients with metabolic syndrome are older, have multiple triggers, longer history of migraine as well as duration of migraine attack. A larger controlled study is needed to confirm these results.

Acknowledgments We thank Rupali Mishra for secretarial help.

Conflict of interest There is no conflict of interest.

Ethics approval The research has been approved by the Institutional Ethics Committee, SGPGIMS, Lucknow.

Open Access This article is distributed under the terms of the Creative Commons Attribution License which permits any use, distribution, and reproduction in any medium, provided the original author(s) and the source are credited.

\section{References}

1. Robbins MS, Lipton RB (2010) The epidemiology of primary headache disorder. Semin Neurol 30:107-119

2. Brennum J, Brinck T, Schriver L et al (1996) Sumatriptan has no clinically relevant effect in the treatment of episodic tension type headache. Eur J Neurol 3:23-28

3. Stang PE, Carson AP, Rose KM et al (2005) Headache, cerebrovascular symptoms, and stroke: the atherosclerosis risk in communities study. Neurology 64:1573-1577

4. Lipton RB, Stewart WF, Cady R et al (2000) Wolfe Award. Sumatriptan for the range of headaches in migraine sufferers: results of the Spectrum Study. Headache 40:783-791

5. Lj Stovner, Hagen K, Jensen R et al (2007) The global burden of headache: a documentation of headache prevalence and disability worldwide. Cephalalgia 27:193-210

6. Sakai F, Igarashi H (1997) Prevalence of migraine in Japan: a nationwide survey. Cephalalgia 17:15-22

7. Horev A, Wirguin I, Lantsberg L, Ifergane G (2005) A high incidence of migraine with aura among morbidly obese women. Headache 5:936-938

8. Rainero I, Limone P, Ferrero M et al (2005) Insulin sensitivity is impaired in patients with migraine. Cephalalgia 25:593-597

9. Guldiken B, Guldiken S, Taskiran B et al (2009) Migraine in metabolic syndrome. Neurologist 15:55-58

10. Bigal E, Liberman JN, Lipton RB (2006) Obesity and migraine: a population study. Neurology 66:545-550

11. Mattsson $P$ (2007) Migraine headache and obesity in women aged 40-74 years: a population-based study. Cephalalgia 27:877-880
12. Takeshima $T$ (2009) Metabolic syndrome and prevention of migraine headache. Brain Nerve 61:1143-1153

13. Guldiken B, Guldiken S, Demir M et al (2008) Insulin resistance and high sensitivity $\mathrm{C}$-reactive protein in migraine. Can J Neurol Sci 35:448-451

14. Cavestro C, Rosatello A, Micca GM et al (2007) Insulin metabolism is altered in migraineurs: a new pathogenic mechanism for migraine? Headache 47:1436-1442

15. Rao NS, Pearce J (1971) Hypothalamic-pituitary-adrenal axis studies in migraine with special reference to insulin sensitivity. Brain 94:289-298

16. Headache Classification Sub-Committee of the International Headache Society (2004) International classification of headache disorders, 2nd edition. Cephalalgia 24:1-160

17. National Cholesterol Education Program (NCEP) Expert Panel on Detection, Evaluation, and Treatment of High Blood Cholesterol in Adults (Adult Treatment Panel III) (2002) Third report of the National Cholesterol Education Program (NCEP) Expert Panel on detection, evaluation, and treatment of high blood cholesterol in adults (Adult Treatment Panel III) final report. Circulation 106: 3143-3421

18. Alberti KG, Alberti KG, Zimmet P, Shaw J, IDF Epidemiology Task Force Consensus Group (2005) The metabolic syndrome-a new worldwide definition. Lancet 366:1059-1062

19. Mattews DR, Hosker JP, Rudenski AS et al (1985) Homeostasis model assessment: insulin resistance and beta cell function from fasting plasma glucose and insulin concentrations in man. Diabetologia 28:412-419

20. Esteghamati A, Ashraf H, Khalilzadeh O et al (2010) Optimal cut-off of homeostasis model assessment of insulin resistance (HOMA-IR) for the diagnosis of metabolic syndrome: third national surveillance of risk factors of non-communicable diseases in Iran (SuRFNCD-2007). Nutr Metab (Lond) 7:26

21. Schele R, Ahlborg B, Ekbom K (1978) Physical characteristics and allergic history in young men with migraine and other headaches. Headache 1:80-86

22. Ford ES, Li C, Pearson WS, Zhao G, Strine TW, Mokdad AH (2008) Body mass index and headaches: findings from a national sample of US adults. Cephalalgia 28:1270-1276

23. Vega GL (2010) Is intra-abdominal obesity a unique risk factor for metabolic syndrome in non-diabetics? Indian J Med Res 131:603-605

24. Winter AC, Berger K, Buring JE, Kurth T (2009) Body mass index, migraine, migraine frequency, and migraine features in women. Cephalalgia 29:269-278

25. Bic Z, Blix GG, Hopp HP, Leslie FM (1998) In search of the ideal treatment for migraine headache. Med Hypotheses 50:1-7

26. Scher AI, Terwindt GM, Picavet HS et al (2005) Cardiovascular risk factors and migraine: the GEM population-based study. Neurology 64:614-620

27. Yadav RK, Kalita J, Misra UK (2010) A study of triggers of migraine in India. Pain Med 11:44-47

28. Masters JB, Mortimer MJ, Hay KM (1986) Glucose and diet in fasting migraineurs. Headache 26:243-247

29. Dexter JD, Roberts J, Byer JA (1978) The five hour glucose tolerance test and effect of low sucrose diet in migraine. Headache 18:91-94

30. Split W, Szydlowska M (1997) Headaches in non insulindependent diabetes mellitus. Funct Neurol 12:327-332

31. Yadav S, Boddula R, Genitta G et al (2008) Prevalence and risk factors of pre-hypertension and hypertension in an affluent north Indian population. Indian J Med Res 128:712-720 